\title{
Comparison of dexmedetomidine and fentanyl as adjuvants to intrathecal levobupivacaine in lower segment cesarean section: A prospective, randomized double blind study
}

\author{
Kapil Rastogi ${ }^{1}$, Alok Kumar Bharti ${ }^{2}$, Yashpal Singh ${ }^{3}$, Pushkar Ranjan ${ }^{4}$ \\ 1-Assistant Professor, Dept. of Anesthesiology, Integral Institute of Medical Science and Research, Lucknow, UP, India. \\ 2-Assistant Professor, Dept. of Anesthesiology and Critical Care, Indira Gandhi Institute of Medical Sciences (IGIMS), Patna, Bihar-800013, India. \\ 3-Associate Professor, Dept of Anesthesiology, AlIMS. Gorakhpur, UP, India. \\ 4-Professor, Dept, of Anesthesiology, IMS, BHU, Varanasi. \\ Correspondence: Dr Alok Kumar Bharti; E-mail: alok.bharti48@gmail.com; Mobile: +91 9307645596
}

\section{Abstract}

Background: Intrathecal bupivacaine is the most commonly used local anesthetic for lower segment cesarean section (LSCS) but there is constant endeavor for search of a local anesthetic (LA) which has improved safety profile for mother as well as the fetus. So far, many adjuvants like fentanyl, morphine or tramadol etc. have been used to prolong intraoperative anesthesia and postoperative analgesia. But the literature lacks information on the use of dexmedetomidine as adjuvants with isobaric levobupivacaine. So, we planned this study to compare dexmedetomidine and fentanyl added to $0.5 \%$ isobaric intrathecal levobupivacaine in spinal anesthesia for LSCS.

Methodology: After institutional ethical committee approval and informed written consent, the patients were divided into three equal groups: Group L; to receive $2.5 \mathrm{ml}$ of isobaric levobupivacaine $0.5 \%$, Group LD to receive $2.5 \mathrm{ml}$ of isobaric levobupivacaine and $5 \mu \mathrm{g}$ dexmedetomidine and Group LF to receive $2.5 \mathrm{ml}$ of isobaric levobupivacaine and $25 \mu \mathrm{g}$ fentanyl intrathecally. Primary outcome was measured as duration of sensory and motor blockade from the time of intrathecal administered drugs. Statistical analysis was performed by using chi-square test or Fischer's exact test and One-way ANOVA or Kruskal Wallis test as applicable. A p-value of $<0.05$ was considered as statistically significant.

Results: Duration of sensory and motor blockade was significantly prolonged $(p<0.001)$ in Group LD as compared to Group LF or L. Onset of sensory and motor blockade was earlier in Group LF as compared to Group LD and L $(p<0.001)$. Time to first rescue analgesia was prolonged in Group LD than Group LF and L $(p<0.001)$.

Conclusion: Intrathecal dexmedetomidine produces prolonged motor blockade as well as postoperative analgesia than fentanyl when used as an adjuvant to $0.5 \%$ isobaric levobupivacaine in elective cesarean section.

Key words: Cesarean section; Dexmedetomidine; Fentanyl; Intrathecal analgesia; Levobupivacaine; Spinal anesthesia

Citation: Rastogi K, BhartiAK, Singh Y, Ranjan P. Comparison of dexmedetomidine and fentanyl as adjuvants to intrathecal levobupivacaine in lower segment cesarean section: A prospective, randomized double blind study. Anaesth. pain intensive care 2020;24(3):383-388.

Received: 18 April 2020, Reviewed: 14 May 2020, Revised: 15 May 2020, Accepted: 23 June 2020

\section{Introduction}

Subarachnoid block is the most widely used regional anesthetic procedure for lower abdominal and lower limb surgery including lower segment cesarean section
(LSCS). ${ }^{1}$ It provide rapid onset, consistent sensory and motor blockade with adequate muscle relaxation for all types of surgery below the level of umbilicus.

Regional anesthesia is safer than general anesthesia and has the advantage of the parturient being awake during the 
birth process. ${ }^{2}$ Intrathecal local anesthetics (LA) alone are not enough for effective postoperative analgesia and higher doses of LA are also associated with hemodynamic instability, which can lead to unfavorable maternal and fetal outcome. So far many adjuncts have been used to augment the analgesia produced by intrathecal LA and to reduce their adverse effects. ${ }^{3}$ The very latest addition to LA used for labor analgesia is levobupivacaine, which is the left-turning molecule of bupivacaine. ${ }^{4}$ Levobupivacaine seems identical to bupivacaine in potency and shows longer duration of action on neural tissue. Because bupivacaine is a racemic mixture of both the left- and rightturning molecules, it has been recently referred to as racemic bupivacaine to further distinguish it from levobupivacaine. $^{4}$ Isobaric levobupivacaine has less cardiotoxicity propensity and less chance of cephalic spread, which favor particularly in LSCS.

It has been well documented that a combination of fentanyl and LA administered intrathecally has synergistic analgesic effects. ${ }^{5}$ Fentanyl is a lipophilic $\mu$-receptor agonist opioid. Intrathecally, fentanyl exerts its effect by combining with opioid receptors in the dorsal horn of spinal cord and may have a supra spinal spread and action. Dexmedetomidine is a highly selective $\alpha 2$-adrenoceptor agonist, and recently used as an adjuvant with intrathecal LA and found to prolong the duration of sensory and motor blockade as well as to provide hemodynamic stability during intraoperative period. ${ }^{6-10}$ It also produces dosedependent sedation, anxiolysis and analgesia (involving spinal and supraspinal sites) without respiratory depression.

Review of literature on use of dexmedetomidine as adjuvants with $0.5 \%$ isobaric intrathecal levobupivacaine in LSCS revealed very little data. So, we planned this study to compare dexmedetomidine with fentanyl as adjuvants to intrathecal isobaric $0.5 \%$ ropivacaine in cesarean sections.

\section{Methodology}

After institutional ethical committee approval and informed written consent, 60 parturients were enrolled in this prospective randomized double blinded controlled study. Inclusion criteria included full term parturients of American Society of Anesthesiology (ASA) grade 2, between ages 20-40 y, scheduled for elective LSCS. Exclusion criteria included patient refusal, having allergy to studied drugs, any contraindication to spinal anesthesia, and pregnancy with associated medical problems like eclampsia, pre-eclampsia, diabetes etc.
All parturients were randomly assigned into three equal groups $(n=20)$ to receive spinal anesthesia: Group L to receive $2.5 \mathrm{ml}$ of isobaric levobupivacaine $0.5 \%$; Group LD to receive $2.5 \mathrm{ml}$ of isobaric levobupivacaine $0.5 \%$ plus $5 \mu \mathrm{g}$ dexmedetomidine and Group LF to receive $2.5 \mathrm{ml}$ of isobaric levobupivacaine $0.5 \%$ plus $25 \mu \mathrm{g}$ fentanyl intrathecally. Each group received a total volume of $3 \mathrm{ml}$. Randomization was performed by anesthesiologist involved in drug preparations. Other investigator involved in the procedure and monitoring was unaware of group allocation. Patients were also blinded to the drug regimen used in spinal anesthesia.

On arrival to operating room, standard monitoring was placed and baseline parameters recorded. Peripheral 18G intravenous (IV) catheter was secured and preloading done with lactated ringer solution $10 \mathrm{ml} / \mathrm{kg}$. Before spinal anesthesia patients were explained about the procedure and methodology of monitoring methods. In the left lateral decubitus position under standard aseptic precautions, using a midline approach lumbar puncture was performed at L3-L4 or L4-L5 intervertebral space by 25G Quincke spinal needle (BD, Gurgaon, Haryana, India). Having confirmed the free flow of cerebrospinal fluid through the spinal needle, the studied drug solution was injected intrathecally over a period of $10-15 \mathrm{sec}$ and patients were turned to the supine position with wedge under right buttock.

Primary outcome included the comparison of the block characteristics and duration of postoperative analgesia. Secondary outcome was to compare the hemodynamic parameters, time to first rescue analgesia and adverse effects of dexmedetomidine or fentanyl given intrathecally with isobaric $0.5 \%$ levobupivacaine.

The level of sensory bock assessed bilaterally in midclavicular line, by loss of pinprick sensation to 23gauge hypodermic needle and dermatomes levels were tested every 2 min until the highest level had stabilized by consecutive tests. The highest dermatome level of sensory blockade, the time to reach this level from the time of injection, time to $\mathrm{S} 1$ level sensory regression was recorded. The motor dermatome level was assessed using Modified Bromage scale. The time to reach Bromage scale 3 before surgery and Bromage 0 in post anesthesia care unit (PACU) was recorded. On achieving T-6 sensory blockade level and Bromage scale 3 surgery was allowed. Sedation was assessed by a modified Ramsay sedation scale. Pain was assessed using VAS (0 to 10) scale at time of incision and at completion of surgery. After surgery patient was 
shifted to PACU and observed for hemodynamic parameters, duration of sensory block, duration of motor block, degree of postoperative analgesia and need of rescue analgesic. Hypotension, defined as a decrease of systolic blood pressure by more than $30 \%$ from baseline or a fall below $90 \mathrm{mmHg}$, was treated with incremental IV boluses of ephedrine $5 \mathrm{mg}$ and IV fluid as required. Bradycardia, defined as heart rate $<50 \mathrm{bpm}$, was treated with IV atropine $0.3-0.6 \mathrm{mg}$. The incidence of adverse effects, such as nausea, vomiting, shivering, pruritus, respiratory depression, sedation, and hypotension was recorded.

Our estimated sample size was based on the study efficacy in term of degree of sensory and motor blockade among the group. For sample size calculation, we defined a relevant clinical difference of $20 \%$ in degree of blockade among the three groups. Thus, sample size of 15 per group with effect size of 20 provided $90 \%$ power for detecting significant differences at any point of time between three groups at alpha level of 0.05 . Sixty patients were randomly allocated into one of the three groups using sealed envelopes based on computer generated random number.

\section{Statistical analysis}

Statistical analysis was done with the Statistical Package for the Social Science (SPSS) version 17.0. Continuous variables are presented as mean $\pm \mathrm{SD}$, and categorical variables are presented as absolute numbers and percentage. The comparison of normally distributed continuous variables between the groups was performed using One-Way ANOVA or Kruskal Wallis test. Nominal categorical data between the groups were compared using Chi-square test or Fischer's exact test. A p-value $<0.05$ was considered as statistically significant.

\section{Results}

Sixty parturients completed the study successfully (Figure 1). The study groups were comparable in terms of demographic profile and baseline parameters (Table 1). Time required to achieve highest level of sensory block was the shortest in Group LF ( $3.35 \pm 0.36 \mathrm{~min})$, maximum in Group L (5.56 $\pm 0.91 \mathrm{~min})$, difference being highly significant between three groups $(\mathrm{p}<0.001)$ (Table 2). Average time required to achieve Bromage scale 3 varied similarly, least in Group LF $(2.94 \pm 0.30)$ and statistically highly significant between the three groups $(\mathrm{p}<0.001)$ (Table 2). The time required for sensory regression to $\mathrm{S} 1$ level (duration of sensory block) was maximum in Group LD $(316.10 \pm 24.34 \mathrm{~min})$ and was highly significant between three groups $(\mathrm{p}<001)$ (Table 2). The time required to reach Bromage scale 0 (duration of motor block) was highest in Group LD followed by LF and L $(265.05 \pm 12.68$ vs. $203.25 \pm 23.80$ vs. $176.00 \pm 18.61 \mathrm{~min})$ and statistically significant between three groups ( $\mathrm{p}<$ 0.001) (Table 2). The differences in time required for first analgesic requirement (duration of analgesia) was highly significant between groups $(\mathrm{p}<0.001)$, being the highest in Group LD (275.75 $\pm 20.21 \mathrm{~min})$ and the lowest in Group $\mathrm{L}(199.00 \pm 18.68 \mathrm{~min}$ ) (Table 2). There were no statistically significant differences in hemodynamic parameters and associated adverse events between the three groups (Table 3).

Figure 1: Comparison of sedation score between groups.

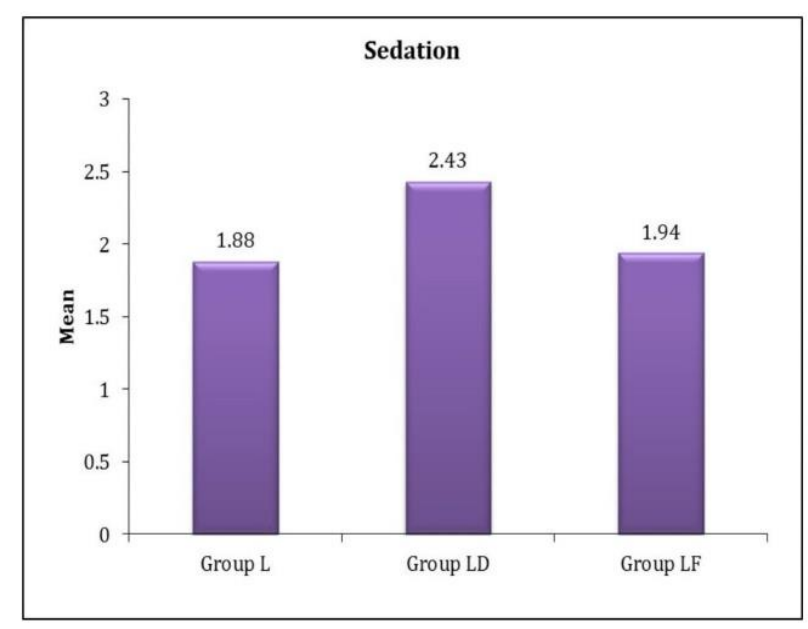

\section{Discussion}

Levobupivacaine is a long acting amide LA and senantiomer of bupivacaine, known to have less chance of cardiotoxicity and less cephalic spread. So, we conducted this trial to study its comparative efficacy when used with adjuvants dexmedetomidine or fentanyl intrathecally for LSCS.

In this present study, mean duration of sensory blockade (time to regression to $\mathrm{S} 1$ dermatome) and motor blockade (time to regression to Bromage scale 0), was found to be prolonged for dexmedetomidine group than the other two groups. This explains that intrathecal dexmedetomidine earlier study by Gupta et al. They studied the analgesic effects of dexmedetomidine $(5 \mu \mathrm{g})$ or fentanyl $(25 \mu \mathrm{g})$ given intrathecally with hyperbaric $0.5 \%$ bupivacaine $(12.5 \mathrm{mg})$, and concluded that intrathecal dexmedetomidine was associated with prolonged motor and sensory block, hemodynamic stability, and reduced 
Table 1: Comparison of demographic data and baseline parameters between the groups. $(n=20)$

\begin{tabular}{lllll}
\hline Parameter & Group L & Group LD & Group LF & P value \\
\hline Age (Year) & $27.10 \pm 4.15$ & $27.10 \pm 3.85$ & $27.75 \pm 3.98$ & $1.00^{* * * * \#}$ \\
\hline Weight (Kg) & $57.00 \pm 5.75$ & $54.20 \pm 4.50$ & $55.55 \pm 5.25$ & $0.28^{*}, 1.00^{* *} . \#$ \\
\hline Height (Cm) & $154.10 \pm 3.50$ & $155.90 \pm 5.79$ & $155.25 \pm 4.26$ & $0.67^{*}, 1.00^{* *}, \#$ \\
\hline BMl & $23.92 \pm 2.18$ & $22.33 \pm 1.70$ & $22.89 \pm 1.82$ & $0.03^{*}, 1.00^{* *}, 0.28^{\#}$ \\
\hline Baseline HR (beats/min) & $85.55 \pm 6.12$ & $85.45 \pm 7.84$ & $82.85 \pm 7.05$ & $0.96^{*}, 0.20^{* *}, 0.28^{\#}$ \\
\hline Baseline MAP (mmHg) & $95.90 \pm 2.19$ & $94.95 \pm 6.37$ & $94.90 \pm 5.04$ & $0.53^{*}, 0.42^{* *}, 0.98^{\#}$
\end{tabular}

Data presented as mean $\pm S D .{ }^{*}$ Group $L$ versus Group $L D,{ }^{* *}$ Group $L$ versus Group $L F,{ }^{*}$ Group $L D$ versus Group $L F . P<0.05$ considered as significant. $S D=$ Standard Deviation, $B M I=$ Body Mass Index, $H R=$ Heart Rate, MAP = Mean Arterial Pressure. Pearson's chi-square test and ANOVA test were used for analysis as required.

Table 2: Comparative block characteristics and time to 1 st analgesic requirements $(n=20)$

\begin{tabular}{|c|c|c|c|c|}
\hline Parameter & Group L & Group LD & Group LF & P value \\
\hline $\begin{array}{l}\text { Time to reach highest level of } \\
\text { sensory block (minute) }\end{array}$ & $5.56 \pm 0.91$ & $4.49 \pm 0.53$ & $3.35 \pm 0.36$ & $<0.001^{* * * * \#}$ \\
\hline $\begin{array}{l}\text { Time to reach Bromage } 3 \text { before } \\
\text { surgery (Minute) }\end{array}$ & $4.74 \pm 0.87$ & $3.90 \pm 0.48$ & $2.94 \pm 0.30$ & $<0.001^{*, * *, \#}$ \\
\hline $\begin{array}{l}\text { Time to S1 level sensory } \\
\text { regression (Minute) }\end{array}$ & $189.25 \pm 22.20$ & $316.10 \pm 24.34$ & $244.25 \pm 25.09$ & $<0.001^{* * *, \#}$ \\
\hline $\begin{array}{l}\text { Time to reach Bromage } 0 \text { after } \\
\text { surgery (minute) }\end{array}$ & $176.00 \pm 18.61$ & $265.05 \pm 12.68$ & $203.25 \pm 23.80$ & $<0.001^{* * * * \#}$ \\
\hline $\begin{array}{l}\text { Time of first analgesic } \\
\text { requirements (minutes) }\end{array}$ & $199.00 \pm 18.68$ & $275.75 \pm 20.21$ & $244.00 \pm 16.67$ & $<0.001^{* * * * \#}$ \\
\hline
\end{tabular}

Table 3: Comparative frequency of side effects between three groups. $(n=20)$

\begin{tabular}{lllll}
\hline Parameter & Group L & Group LD & Group LF & P value \\
\hline Hypotension & $2(10)$ & $3(15)$ & $3(15)$ & 0.86 \\
\hline Nausea/Vomiting & $2(10)$ & $1(5)$ & $3(15)$ & 0.57 \\
\hline Shivering & $2(10)$ & $0(0)$ & $1(5)$ & 0.35 \\
\hline Reparatory depression & $0(0)$ & $0(0)$ & $2(10)$ & 0.13
\end{tabular}

Data presented as mean $\pm S D$ or number or percentage. *Group $L$ versus Group $L D,{ }^{* *}$ Group $L$ versus Group $L F$, \# Group $L D$ versus Group LF. $P<0.05$ considered as significant. SD = Standard Deviation. 
demand for rescue analgesics in $24 \mathrm{~h}$ as compared to fentanyl. ${ }^{11}$ Our study is further strengthened by a study by Ramadan et al., which evaluated the effects of adding dexmedetomidine $(5 \mu \mathrm{g})$ versus fentanyl $(25 \mu \mathrm{g})$ to intrathecal bupivacaine $(10 \mathrm{mg})$, and concluded that the dexmedetomidine group had significantly longer sensory and motor block times than fentanyl group or control groups. No adverse effects on mothers or babies were noticed among three groups. ${ }^{12}$

How dexmedetomidine prolongs the sensory and motor blockade is not known exactly. Best possible mechanism may be the analgesia produced by $\alpha 2$ agonists occurs as a result of decreased release of C-fiber transmitters and hyperpolarization of postsynaptic dorsal horn neurons. It has been postulated that binding of $\alpha 2$ agonist agents to the dorsal horn motor neurons results in the prolongation of motor blockade of LA. ${ }^{13,14}$ They also exhibit anestheticsparing and hemodynamic- stabilizing effects. ${ }^{15,16}$

In our study. mean onset time of sensory and motor blockade was significantly shorter in Group LF than Group LD or Group L. Contrary to our finding, other studies report that time of onset of sensory and motor blockade was earlier in dexmedetomidine group than fentanyl group. ${ }^{17,18}$ The differences in sensory and motor onset time could be due to the use of isobaric levobupivacaine $(0.5 \%)$, volume of the used diluent, rapidity of intrathecal injection and more lipophilic nature of fentanyl.

Prolonged time to first analgesic requirement in our study in Group LD is supported by a study done by Rahimzadeh et al. they studied the comparative addition of dexmedetomidine $(5 \mu \mathrm{g})$ and fentanyl $(25 \mu \mathrm{g})$ to intrathecal $2.5 \mathrm{ml}$ bupivacaine $0.5 \%$ in orthopedic procedure in lower limbs and concluded that first rescue analgesic request was prolonged in dexmedetomidine group than fentanyl group. ${ }^{19}$

The sedation scores of patients in dexmedetomidine group were significantly better than in the other two groups. This clearly shows that intrathecal dexmedetomidine provides better sedation to patients than intrathecal fentanyl, which can be very useful in patients undergoing cesarean section. In all three groups, the newborns had no signs of fetal distress, as evidenced by an Apgar score $\geq 7$ at $1 \mathrm{~min}$, which infers the advantageous use of dexmedetomidine over other adjuvants and similar results were supported by other studies. ${ }^{20-21}$ The incidence of side effects like nausea and vomiting, hypotension, bradycardia, respiratory depression, shivering and pruritus were not significantly different among the groups.

\section{Limitations}

As the present study contributes to the existing knowledge on $\alpha 2$ agonists, certain limitations must be taken into consideration. All the patients included in the study were ASA physical status II; as such caution must be exerted while generalizing the results to ASA physical status III and IV patients. It was conducted on patients scheduled for LSCS and it is possible that the level of surgery might alter the perception of post-operative pain. Therefore, further clinical studies are needed to determine the equivalent doses of dexmedetomidine and fentanyl for different types of neuraxial blockade. We did not compare 24-hour analgesic requirements between the groups.

\section{Conclusion}

We conclude that $5 \mu \mathrm{g}$ dexmedetomidine is better alternative to $25 \mu \mathrm{g}$ fentanyl as an adjuvant to intrathecal levobupivacaine in cesarean section. It provides early sensory and motor block, prolonged intraoperative and postoperative analgesia, sedation, hemodynamic stability, minimal side effects, and no adverse effect on Apgar scores of newborns.

\section{Conflict of Interest}

None

\section{Authors' contribution}

KR: Content of work, Data collection

AKB: Concept, manuscript writing, editing

YS: Data analysis and interpretation

PR: Study design

\section{References}

1. Hinnerk FWW. The centennial of spinal anaesthesia. Anesthesiology. 1998;89:500-506. [PubMed] DOI: 10.1097/00000542-199808000-00028

2. Hawkins JL, Koonin LM, Palmer SK, Gibbs CP. Anesthesia - related deaths during obstetric delivery in the United States, 1979-1990. Anesthesiology. 1997;86:277-284. [PubMed] DOI: 10.1097/00000542199702000-00002

3. Yu SC, Ngan Kee WD, Kwan ASK. Addition of meperidine to bupivacaine for spinal anesthesia for 
Caesarean section. Br J Anaesth. 2002;88:379-383. [PubMed] DOI: 10.1093/bja/88.3.379

4. McLeod GA, Burke D. Levobupivacaine. Anaesthesia. 2001;56:331-341. [PubMed] DOI: 10.1046/i.13652044.2001.01964.x

5. Wang C, Chakrabarti MK, Whitwam JG. Specific enhancement by fentanyl of the effects of intrathecal bupivacaine on nociceptive afferent but not on sympathetic efferent pathways in dogs. Anesthesiology .1993;79:766-773. [PubMed] DOI: 10.1097/00000542199310000-00019

6. Singh AK, Singh $Y$, Jain G, Verma RK. Comparison of two different doses of intrathecal dexmedetomidine as adjuvant with isobaric ropivacaine in lower abdominal surgery. Anesth Essays Res. 2015;9:343-347. [PubMed] DOI: $10.4103 / 0259-1162.158009$

7. Scafati A. Analgesia and alpha agonists 2. Medens Rev. 2004;4.

8. Vieira AM, Schnaider TB, Brandão AC, Pereira FA, Costa ED, Fonseca CE. Epidural clonidine or dexmedetomidine for post-cholecystectomy analgesia and sedation. Rev Bras Anestesiol. 2004 Aug;54(4):473-478. [PubMed] DOI: 10.1590/s003470942004000400003

9. Hall JE, Uhrich TD, Barney JA, Arain SR, Ebert TJ. Sedative, amnestic, and analgesic properties of smalldose dexmedetomidine infusions. Anaesth Analg. 2000;90:699-705. [PubMed] DOI: 10.1097/00000539200003000-00035

10. Scheinin $M$, Pihlavisto M. Molecular pharmacology of alpha 2-adrenoceptor agonists. Bailliere's Clin Anaesth. 2000;14:247-60.

11. Gupta R, Verma R, Bogra R, Kohli M, Raman R, Kushwaha JK. A comparative study of intrathecal dexmedetomidine and fentanyl as adjuvant to bupivacaine. J Anaesthesiol Clin Pharmacol. 2011;27:339-343. [PubMed] DOI: 10.4103/0970$\underline{9185.83678}$

12. Mahdy WR, Sabry IA. Effect of adding dexmedetomidine versus fentanyl to intrathecal bupivacaine on spinal block characteristics and neonatal outcome in uncomplicated cesarean: a randomized double blind placebo controlled study. Menoufiya Med J. 2011;24(1):221-232. [Free Full Text]
13. Kanazi GE, Aouad MT, Jabbour-Khoury SI, Al Jazzar $\mathrm{MD}$, Alameddine MM, Al-Yaman R, et al. Effect of low dose dexmedetomidine or clonidine on the characteristics of bupivacaine spinal block. Acta Anaesthesiol Scand. 2006;50:222-227. [PubMed] DOI: 10.1111/j.1399-6576.2006.00919.x

14. Bajwa SJ, Bajwa SK, Kaur J, Singh G, Arora V, Gupta $S$, et al. Dexmedetomidine and clonidine in epidural anaesthesia: A comparative evaluation. Indian J Anaesth. 2011;55:116-121. [PubMed] DOI: 10.4103/0019-5049.79883

15. Gertler R, Brown HC, Mitchell DH, Silvius EN. Dexmedetomidine: A novel sedative analgesic agent. Proc (Bayl Univ Med Cent) 2001;14:13-21. [PubMed] DOI: 10.1080/08998280.2001.11927725

16. Paris $\mathrm{A}$, Tonner PH. Dexmedetomidine in anaesthesia. Curr Opin Anaesthesiol. 2005;18:412-418. [PubMed] DOI: $\underline{10.1097 / 01 . a c 0.0000174958 .05383 . d 5}$

17. Yektas A, Belli E. The effects of $2 \mu \mathrm{g}$ and $4 \mu \mathrm{g}$ doses of dexmedetomidine in combination with intrathecal hyperbaric bupivacaine on spinal anesthesia and its postoperative analgesic characteristics. Pain Res Manag. 2014;19:75-81. [PubMed] DOI: 10.1155/2014/956825

18. Ravipati P, Isaac GA, Reddy PN, Krishna L, Supritha T. A comparative study between intrathecal isobaric Ropivacaine $0.75 \%$ plus Dexmedetomidine and isobaric Ropivacaine $0.75 \%$ plus fentanyl for lower limb surgeries. Anesth Essays Res. 2017;11:621-626. [PubMed] DOI: 10.4103/0259-1162.206857

19. Rahimzadeh $P$, Faiz SH, Imani $F$, Derakhshan $P$, Amniati S. Comparative addition of dexmedetomidine and fentanyl to intrathecal bupivacaine in orthopedic procedure in lower limbs. BMC Anesthesiology 2018;18:62. [PubMed] DOI: 10.1186/s12871-018-0531?

20. Sun $Y, X u Y$, Wang $G N$. Comparative evaluation of intrathecal bupivacaine alone, bubivacaine-fentanyl, and bupivacaine-dexmedetomidine in cesarean section. Drug Res (Stuttg). 2015;65:468-472. [PubMed] DOI: 10.1055/s-0034-1387740

21. Paech MJ, Pavy TJ, Orlikowski CE, Yeo ST, Banks SL, Evans SF, et al. Postcesarean analgesia with spinal morphine, clonidine, or their combination. Anesth Analg. 2004;98:1460-1466. [PubMed] DOI: 10.1213/01.ane.0000111208.08867.3c 